\title{
The University of Michigan Specialist-Hospitalist Allied Research Program: Jumpstarting Hospital Medicine Research
}

\author{
Scott A. Flanders, $\mathrm{MD}^{1,2}$ \\ Samuel R. Kaufman, $\mathrm{MA}^{2,3}$ \\ Brahmajee K. Nallamothu, MD, MPH ${ }^{4,5}$ \\ Sanjay Saint, MD, MPH ${ }^{4,1-3}$ \\ ${ }^{1}$ Hospitalist Program, Division of General Medi- \\ cine, Department of Internal Medicine, University \\ of Michigan, Ann Arbor, Michigan \\ ${ }^{2}$ Division of General Medicine, Department of In- \\ ternal Medicine, University of Michigan, Ann \\ Arbor, Michigan \\ ${ }^{3}$ VA/UM Patient Safety Enhancement Program, \\ Ann Arbor, Michigan \\ ${ }^{4}$ Center for Practice Management and Outcomes \\ Research, Ann Arbor VA Health Services \\ Research \& Development Center of Excellence, \\ Ann Arbor, Michigan \\ ${ }^{5}$ Division of Cardiology, Department of Internal \\ Medicine, University of Michigan, Ann Arbor, \\ Michigan
}

BACKGROUND: Clinical research has developed slowly in most academic hospitalist programs, possibly because of a failure to recognize the important role of specialists in the diagnosis and management of complex medical patients as well as their expertise in clinical research. Ideally, a successful hospital-based clinical research program will need to partner hospitalists with specialists.

PURPOSE: The University of Michigan's Specialist-Hospitalist Allied Research Program (SHARP) was designed to jumpstart hospital-based clinical and translational research at a major academic medical center by pairing specialists and hospitalists to ask and answer novel research questions.

DESCRIPTION: SHARP is codirected by a hospitalist and a subspecialist and includes key personnel such as a hospitalist investigator, a clinical research nurse, a research associate, and a clinical epidemiologist. The program is guided by an oversight committee that includes institutional research leadership. Two initial projects have already been supported. The first, a collaboration between infectious disease specialists and hospitalists, is a prospective trial of antiseptic agents and techniques to reduce false-positive blood cultures. The second pairs geriatricians and clinical pharmacists with hospitalists to prospectively study techniques to reduce medication errors around the time of hospital discharge. Although initial pilot projects are single-institution studies, SHARP's goal is to expand its clinical research to include multicenter investigation. Metrics to evaluate SHARP include the number of successfully completed projects, extramural grants submitted and funded, and peer-reviewed publications.

CONCLUSION: A successful hospital-based clinical research program combines hospitalists and specialists in a collaborative environment to identify optimal strategies for delivering inpatient care. Journal of Hospital Medicine 2008;3:308313. () 2008 Society of Hospital Medicine.

KEYWORDS: hospitalist, interdisciplinary research, research skills, specialist.

D ramatic changes in the organization, financing, and delivery of hospital care that began a decade ago continue to accelerate. One of the most important changes has been the emergence of hospitalists as providers of inpatient care. ${ }^{1}$ Hospitalists are physicians, usually general internists, whose clinical focus is the hospitalized patient. As patient illnesses have become more severe and complex, physicians have found it difficult to balance inpatient and outpatient care and have focused on one of the two. ${ }^{2-5}$ It is estimated that there are currently 15,000 practicing hospitalists nationally, and projections suggest that this number may exceed 30,000 by 2010 , which is equal to the number of cardiologists currently practicing in the United States. ${ }^{6}$ A 2003
The authors thank Dr. Marc E. Lippman, Dr. Robert F. Todd, Dr. Larry McMahon, Dr. Timothy J. Laing, and Mr. Lindsay J. Graham, whose support made this program possible.

This article was presented in part at the Society of Hospital Medicine Annual Meeting, Dallas, TX, May 2007. 
survey from the American Hospital Association showed that more than $30 \%$ of the nation's 4900 community hospitals have hospital medicine groups. ${ }^{7}$ Furthermore, more than $70 \%$ of the nation's largest hospitals ( $>500$ beds) and $66 \%$ of major teaching hospitals use hospitalists. ${ }^{7}$

The transition to a hospitalist model generates multiple new research questions about the best approach to caring for the hospitalized patient. Additionally, hospitalists may spawn new areas of clinical research by tackling clinical issues that formerly lacked a large number of specialist investigators. Examples include implementation-based studies, ${ }^{8,9}$ inpatient safety practices, ${ }^{10-12}$ quasi-experimental studies focusing on common inpatient issues, ${ }^{13,14}$ and the evaluation of new methods for reducing resource utilization within various inpatient care delivery structures. ${ }^{15,16}$

Similarly, if future clinical trials are to be carried out in real-world settings, by necessity these will require the participation of hospitalists. Clinical research performed by hospitalists and hospital medicine programs, however, remains underdeveloped. Although this has been attributed to several variables, including the youth of the field, a paucity of fellowship-trained hospitalist researchers, and a lack of a hospitalist-oriented national funding source, we also believe that additional barriers exist which could be overcome if hospitalists actively partnered with specialists to perform hospital-based clinical and translational research.

Hospitalists lack clinical expertise in many clinical issues. In both academic and nonacademic settings, the diagnostic approach, individual treatment decisions, and follow-up of complex patients occur with frequent consultation of specialists. Specialists often provide a deeper understanding of both the pathophysiologic concepts and scientific principles underlying important clinical questions and are more likely to have had fellowship training that included clinical research experience. Specialists also have more access to extramural funding for disease-based investigation, and thus their involvement in hospital-based clinical research would likely enhance funding opportunities, improve project feasibility, and increase dissemination of the results. A successful clinical research program will therefore be one that combines specialists and hospitalists working collaboratively to determine the best way to care for inpatients. With that in mind, we cre- ated the University of Michigan Specialist-Hospitalist Allied Research Program (SHARP).

\section{METHODS \\ Setting}

The University of Michigan Medical Center includes a 900-bed teaching hospital with more than 44,000 yearly inpatient discharges, and the Department of Internal Medicine manages nearly 15,000 annual discharges. The University of Michigan Hospital Medicine Program has grown dramatically over the past few years and now includes more than 30 hospitalists. These hospitalists will manage nearly 8000 admissions in the upcoming year, which represent more than half of all the patients admitted to the Department of Internal Medicine. Five years ago, these 8000 admissions would have been cared for by 3 to 4 times as many providers, most of whom would have been specialists. Currently, specialists consult regularly on patients cared for by hospitalists, and as a result, a few loosely formed research collaborations developed spontaneously but lacked resources or infrastructure to facilitate their completion. SHARP was intended to organize these clinical research pilot studies and jumpstart hospital-based clinical and translational research.

\section{The SHARP Intervention Objectives}

In 2006, hospitalists and specialists with an interest in expanding clinical and translational research aimed at caring for inpatients were brought together for the SHARP intervention. This intervention had several objectives:

1. To develop a clinical research infrastructure within the University of Michigan Hospital Medicine Program to facilitate patient participation.

2. To foster increased specialist-hospitalist collaboration for addressing common inpatient problems.

3. To facilitate pilot projects and preliminary data collection that enhance the ability to obtain subsequent extramural funding for collaborative research projects.

4. To facilitate multicenter investigation led by the University of Michigan by allowing the SHARP investigators to use an existing hospitalist consortium to expand the scope of research projects.

5. Ultimately, to develop the ability to perform multicenter intervention-based clinical trials. 


\section{Structure}

The key to SHARP's infrastructure is its personnel and governance structure. At the head of SHARP is an academic hospitalist as principal investigator (PI) and an academic cardiologist with health services research training serving as coprincipal investigator (Co-PI). Key personnel also include a hospitalist investigator, a masters-level research associate, a $\mathrm{PhD}$ clinical epidemiologist, and the hospitalists and subspecialists who serve as investigators. Although the program leadership has research experience, many of the hospitalist and specialist investigators are junior faculty without extensive prior research experience. Thus, SHARP was specifically designed to build the capacity to enhance inpatient clinical and translational research and to remove barriers for new investigators developing their academic careers.

It is critical that oversight provides direction for the research program, assists with project identification and selection, and facilitates collaborations that tie diverse projects together. We believe that this is best accomplished by the creation of a steering committee chaired by both the PI and Co-PI. The steering committee also includes key individuals such as the Vice Chair of the Department of Medicine and the Associate Dean for Clinical and Translational Research at the University of Michigan. The 2 cochairs are responsible for overseeing the program and reporting the progress of SHARP to the University of Michigan Department of Internal Medicine. They will help identify and produce viable research proposals that can be brought to the full committee. To help the program understand and overcome bureaucratic obstacles, we have also included a former high-level administrator on the steering committee as a consultant. Given the initial scope of the program, the SHARP steering committee has had a small number of key individuals. As the program grows and increases its number of ongoing collaborative projects, we will likely need to expand committee membership.

SHARP leadership meets regularly to plan projects, discuss grant ideas, make hiring decisions, and troubleshoot problems in existing projects. The entire steering committee meets quarterly to help chart the overall course of the program. A more thorough description of the program and its structure can be found on the SHARP Web site (www.med.umich.edu/sharp).

\section{SHARP Funding}

SHARP could not exist without resources. The funding for the program comes from the Department of Internal Medicine and uses revenue from the hospital medicine program that flows to the department. To garner support for the program, SHARP leadership sought buy-in from the Chair of Medicine, all the division chiefs, and key faculty active in clinical research. The fact that the program has the potential to benefit not just hospitalists but also other department faculty such as specialists facilitated departmental funding. The program is funded for 3 years with an 18-month program review to gauge progress. Funding is used to build clinical research infrastructure and facilitate collection of pilot data. SHARP resources support a portion of the salaries of key personnel for the 3-year duration of the project (research associate, $50 \%$; PI, $10 \%$; Co-PI, $5 \%$; and epidemiologist, 5\%), after which time intramural funding ends. Every SHARP project is, therefore, expected to apply for extramural funding with the goal of full extramural programmatic support after 3 years.

\section{SHARP Performance Metrics}

Measuring the accomplishments of SHARP is clearly important. As the program is intended to jumpstart collaborative inpatient clinical research, the number of such projects is important to track. An additional goal is to support work that leads to extramural funding. As the program started from "scratch," it is unrealistic to have completed peerreviewed manuscripts or successful extramural grants as the sole metrics by which the program is judged, especially early in its initiation. In a yearly report to the department chair, we will report on primary and secondary outcomes (see Table 1).

\section{Initial SHARP Projects}

SHARP has a formal process for evaluating potential projects. A steering committee ultimately decides how best to use SHARP-related resources. Key components in this decision are related to the proposal's innovation, feasibility, and importance as well as the extent of specialist-hospitalist collaboration. The 2 projects described next are our initial areas of focus and exemplify these concepts. One project partners hospitalists with infectious disease specialists, whereas the second pairs 
TABLE 1

Primary and Secondary Outcomes of SHARP

Primary outcomes

1. Number of ongoing research projects involving SHARP support and a brief description of the aims and status of each

2. Number of extramural grants submitted in which SHARP is mentioned or involved

3. Extramural grants received (total and direct dollars)

4. Peer-reviewed publications authored by SHARP investigators

Secondary outcomes

1. Abstracts accepted for presentation at national or international scientific meetings

2. Non-peer-reviewed publications related to SHARP

3. Invited presentations by SHARP investigators

4. People who have visited the University of Michigan in conjunction with SHARP work (eg, visiting professors)

Abbreviation: SHARP, Specialist-Hospitalist Allied Research Program.

hospitalists with geriatricians and clinical pharmacists.

Reducing False Positive Blood Cultures. The blood culture is an important tool for the diagnosis and management of bloodstream infections. As a result, physicians have a low threshold for obtaining blood cultures. Unfortunately, up to half of all positive blood cultures are positive because of contamination. These false positive cultures lead to additional diagnostic testing, unnecessary antibiotics, and increased healthcare costs. ${ }^{17}$ A variety of antiseptic agents and techniques are used to prevent falsely positive cultures. However, a recent evidence-based systematic review performed by University of Michigan investigators found no clear evidence to suggest which antiseptic agent should be routinely used. They concluded that a randomized controlled trial was urgently needed. ${ }^{18}$

SHARP and its infrastructure have begun a cluster-randomized crossover trial at the university hospital. The trial compares the effects of a variety of skin antiseptic agents on peripheral blood culture contamination rates. The study population includes hospitalized patients undergoing venipuncture for peripheral blood cultures on 3 general medicine and surgery floors. The trial will include over 12,000 blood culture sets and will have $85 \%$ power to detect a $0.5 \%$ difference in effectiveness between antiseptic agents. Key outcomes will be rates of positive blood cultures (true positive versus false positive), quantity of additional diagnostic testing generated by positive cultures, resource use (including antibiotics), and associated costs. Clinical outcomes such as length of stay and inpatient mortality will also be measured as secondary outcomes.

Pharmacist-Facilitated Hospital Discharge. Hospital discharge is a complex process in which patients must be transferred from the care of an inpatient team to that of an outpatient provider. During most hospitalizations, a patient will have new medications added, a chronic medication stopped, or a change in medication dosage. Studies have revealed that the most common adverse events that have an impact on patients after discharge are related to medications. ${ }^{19-21}$ In our experience at the University of Michigan, patients frequently have medication-related adverse events after discharge because they do not understand what medications they should be taking, what they are used for, how to manage side effects, or whom to call with problems. In addition, predictable medication-related issues (such as the ability to pay for a medicine or expected serum electrolyte changes with newly added medications) are not universally anticipated. The frail elderly are especially vulnerable to medication-related adverse events.

Building on the work of others in the field, we proposed studying the impact of an inpatient clinical pharmacist to address medication misadventures related to hospital discharge in our elderly population. ${ }^{22}$ The study uses an interrupted time series design (the pharmacist will alternate months at a nonresident hospitalist service and a resident general medicine service) to measure the impact of the clinical pharmacist. The pharmacist will focus on patients over the age of 65 meeting criteria that identify them to be at high risk for an adverse medication event after discharge. These factors include any new medication started in the hospital, medication noncompliance or an adverse medication event that led to the admission, or use of a high-risk medication (eg, anticoagulants, narcotics, diuretics, diabetic agents, and immunosuppressives). The pharmacist and inpatient physicians will identify high-risk patients who will receive predischarge medication counseling. This process will identify problem medications and needed follow-up (eg, laboratory testing) and assess compliance issues. After discharge, patients will be contacted by the pharmacist both within 72 hours and at 30 days. Standardized questions will be asked of patients to troubleshoot medica- 
tion issues, assess them for problems with medications or follow-up, and identify patients who may need more urgent access to a healthcare provider to address medication-related problems.

Key outcomes will include the pharmacist's actions at discharge (eg, dose changes made, medication class switches, and side-effect monitoring implemented). In addition, we will track types of medication issues identified after discharge and interventions made. Important clinical outcomes will include return to the emergency department after discharge, 30-day readmission rates, and healthcare-related costs.

\section{DISCUSSION AND NEXT STEPS}

SHARP is a novel clinical research program partnering hospitalists with specialists. Its current focus targets single-institution studies that generate pilot data leading to larger projects. The ultimate goal is to develop the ability to do larger multicenter investigator-initiated projects. The SHARP program will also have the ability to perform observational studies to identify predictors and risk factors and the ability to carry out implementation studies that show how best to translate results from published articles to direct patient care.

A specialist-hospitalist collaboration overcomes barriers that we feel may impede hospital medicine research at an academic medical center. For a similar program to succeed at other institutions, key components from our program will have to be replicated. First, senior, fellowshiptrained researchers are required to mentor junior investigators (who may or may not have additional fellowship training), help guide project selection, oversee grant and manuscript submissions, and troubleshoot problems that arise in the course of any clinical research project. In our institution, this comes from within our hospitalist program and from our specialist collaborators. In institutions lacking hospitalists with research experience, this guidance could come from within a division of general medicine, internal medicine specialty divisions, internal medicine department leadership, or even non-internal medicine departments (eg, emergency medicine, neurology, and surgery) that have traditionally been involved in clinical research programs.

A second key component that must be considered is funding. An initial investment is necessary to fund key personnel dedicated to getting projects started on the right track, collecting pilot data, and ensuring project completion and dissemination of the results. The positive "margin" generated by our hospitalist program facilitated the initial investment. In the absence of a positive margin, resources could come directly from the hospital, the medical school, the department of internal medicine, or perhaps a foundation. The case would need to be made that an initial shortterm investment would enhance the academic standing of the institution, enhance the careers of young investigators, and over time lead to a selfsustaining program through investigator-initiated grants and extramural funding. In addition to experienced leadership and funding, we created an oversight committee, but we feel that this is not a critical component. A potential concern with a program that partners with specialists might be that research topics become too disease-specific or "specialty-oriented." We specifically created the oversight committee to protect against this possibility, and other institutions might need similar safeguards.

Our next step includes leveraging existing hospitalist collaboratives that reach beyond academic medical centers to expand further the reach of SHARP. Ultimately, any new therapy, clinical tool, diagnostic paradigm, or implementation strategy that is developed or evaluated by the SHARP program would need to be tested in a real-world setting to assess external validity. With support from the Blue Cross Blue Shield of Michigan Foundation, we have created a multihospital patient safety consortium, the Hospitalists as Emerging Leaders in Patient Safety Consortium, which includes academic, government, urban, rural, teaching, and nonteaching hospitals. ${ }^{23}$ Although the initial focus is patient safety, our goal for the consortium is to develop it into a multihospital clinical research program that could take pilot projects developed by SHARP and test them in real-world settings. We believe that full-scale multihospital studies based on SHARP pilot data will be very attractive to external funding agencies and will help SHARP become financially self-sufficient after the initial 3-year start-up.

Hospital medicine research is desperately needed. $^{24,25}$ Unfortunately, the clinical research capabilities of most hospital medicine programs are quite underdeveloped. We believe that part- 
nering hospitalists with specialists can facilitate collaborative research to identify the best way to care for inpatients. If successful, we believe that variations of this model can be replicated at other institutions and will be a critical factor in jumpstarting hospital medicine clinical research.

Address for correspondence and reprint requests: Scott A. Flanders, MD, Department of Internal Medicine, Taubman Center, 1500 East Medical Center Drive, Room 3119F, Ann Arbor, MI 48109-0376; Fax: 734-615-8401; E-mail: flanders@umich.edu

Received 30 July 2007; revision received 21 November 2007; accepted 6 January 2008.

\section{REFERENCES}

1. Wachter RM, Goldman L. The emerging role of "hospitalists" in the American health care system. $N$ Engl J Med. 1996;335:514-517.

2. Saint S, Zemencuk JK, Hayward RA, Golin CE, Konrad TR, Linzer M. What effect does increasing inpatient time have on outpatient-oriented internist satisfaction? J Gen Intern Med. 2003;18:725-729.

3. Saint S, Konrad TR, Golin CE, Welsh D, Linzer M. Characteristics of general internists who practice only outpatient medicine: results from the physician worklife study. Semin Med Pract. 2002;5:5-11.

4. Saint S, Flanders SA. Hospitalists in teaching hospitals: opportunities but not without danger. J Gen Intern Med. 2004;19:392-393.

5. Flanders SA, Wachter RM. Hospitalists: the new model of inpatient medical care in the United States. Eur J Intern Med. 2003;14:65-70.

6. Lurie JD, Miller DP, Lindenauer PK, Wachter RM, Sox HC. The potential size of the hospitalist workforce in the United States. Am J Med. 1999;106:441-445.

7. Kralovec PD, Miller JA, Wellikson L, Huddleton JM. The status of hospital medicine groups in the United States. J Hosp Med. 2006;1:75-80.

8. Krein SL, Olmsted RN, Hofer TP, et al. Translating infection prevention evidence into practice using quantitative and qualitative research. Am J Infect Control. 2006;34: 507-512.

9. Auerbach AD, Wachter RM, Katz P, Showstack J, Baron RB, Goldman L. Implementation of a voluntary hospitalist service at a community teaching hospital: improved clinical efficiency and patient outcomes. Ann Intern Med. 2002; 137:859-865.

10. Shojania KG, Duncan BW, McDonald KM, Wachter RM, Markowitz AJ. Making health care safer: a critical analysis of patient safety practices. Evid Rep Technol Assess (Summ). 2001;(43):i-x,1-668.

11. Shojania KG, Duncan BW, McDonald KM, Wachter RM. Safe but sound: patient safety meets evidence-based medicine. JAMA. 2002;288:508-513.

12. Kaboli PJ, Hoth AB, McClimon BJ, Schnipper JL. Clinical pharmacists and inpatient medical care: a systematic review. Arch Intern Med. 2006;166:955-964.

13. Borschel DM, Chenoweth CE, Kaufman SR, et al. Are antiseptic-coated central venous catheters effective in a realworld setting? Am J Infect Control. 2006;34:388-393.

14. Flanders SA, Dudas V, Kerr K, McCulloch CE, Gonzales R. Effectiveness of ceftriaxone plus doxycycline in the treatment of patients hospitalized with community-acquired pneumonia. J Hosp Med. 2006;1:7-12.

15. Meltzer D, Manning WG, Morrison J, et al. Effects of physician experience on costs and outcomes on an academic general medicine service: results of a trial of hospitalists. Ann Intern Med. 2002;137:866-874.

16. Zemencuk JK, Hofer TP, Hayward RA, Moseley RH, Saint S. What effect does physician "profiling" have on inpatient physician satisfaction and hospital length of stay? BMC Health Serv Res. 2006;6:45.

17. Bates DW, Goldman L, Lee TH. Contaminant blood cultures and resource utilization. The true consequences of false-positive results. JAMA. 1991;265:365-369.

18. Malani A, Trimble K, Parekh V, Chenoweth C, Kaufman S, Saint S. Review of clinical trials of skin antiseptic agents used to reduce blood culture contamination. Infect Control Hosp Epidemiol. 2007;28:892-895.

19. Forster AJ, Murff HJ, Peterson JF, Gandhi TK, Bates DW. The incidence and severity of adverse events affecting patients after discharge from the hospital. Ann Intern Med. 2003;138:161-167.

20. Forster AJ, Murff HJ, Peterson JF, Gandhi TK, Bates DW. Adverse drug events occurring following hospital discharge. J Gen Intern Med. 2005;20:317-323.

21. Forster AJ, Clark HD, Menard A, et al. Adverse events among medical patients after discharge from hospital. CMAJ. 2004;170:345-349.

22. Schnipper JL, Kirwin JL, Cotugno MC, et al. Role of pharmacist counseling in preventing adverse drug events after hospitalization. Arch Intern Med. 2006;166:565-571.

23. Flanders SA, Kaufman SR, Saint S. Hospitalists as emerging leaders in patient safety: targeting a few to affect many. J Patient Saf. 2005;1:78-82.

24. Williams MV. The future of hospital medicine: evolution or revolution? Am J Med. 2004;117:446-450.

25. Ranji SR, Rosenman DJ, Amin AN, Kripalani S. Hospital medicine fellowships: works in progress. Am J Med. 2006; 119:72 e1-7. 